\title{
Quasinormal Modes and Strong Cosmic Censorship
}

\author{
Vitor Cardoso, ${ }^{1,2}$ João L. Costa,${ }^{3,4}$ Kyriakos Destounis, ${ }^{1}$ Peter Hintz, ${ }^{5}$ and Aron Jansen ${ }^{6}$ \\ ${ }^{1}$ CENTRA, Departamento de Física, Instituto Superior Técnico-IST, Universidade de Lisboa-UL, \\ Avenida Rovisco Pais 1, 1049 Lisboa, Portugal \\ ${ }^{2}$ Perimeter Institute for Theoretical Physics, 31 Caroline Street North Waterloo, Ontario N2L 2Y5, Canada \\ ${ }^{3}$ Departamento de Matemática, ISCTE_Instituto Universitário de Lisboa, Av. Forças Armadas 1649-026 Lisboa, Portugal \\ ${ }^{4}$ Center for Mathematical Analysis, Geometry and Dynamical Systems, Instituto Superior Técnico-IST, \\ Universidade de Lisboa-UL, Avenida Rovisco Pais 1, 1049 Lisboa, Portugal \\ ${ }^{5}$ Department of Mathematics, University of California, Berkeley, California 94720-3840, USA \\ ${ }^{6}$ Institute for Theoretical Physics and Center for Extreme Matter and Emergent Phenomena, Utrecht University, \\ 3508 TD Utrecht, The Netherlands
}

(Received 2 November 2017; published 17 January 2018)

\begin{abstract}
The fate of Cauchy horizons, such as those found inside charged black holes, is intrinsically connected to the decay of small perturbations exterior to the event horizon. As such, the validity of the strong cosmic censorship (SCC) conjecture is tied to how effectively the exterior damps fluctuations. Here, we study massless scalar fields in the exterior of Reissner-Nordström-de Sitter black holes. Their decay rates are governed by quasinormal modes of the black hole. We identify three families of modes in these spacetimes: one directly linked to the photon sphere, well described by standard WKB-type tools; another family whose existence and time scale is closely related to the de Sitter horizon; finally, a third family which dominates for near-extremally charged black holes and which is also present in asymptotically flat spacetimes. The last two families of modes seem to have gone unnoticed in the literature. We give a detailed description of linear scalar perturbations of such black holes, and conjecture that SCC is violated in the near extremal regime.
\end{abstract}

DOI: 10.1103/PhysRevLett.120.031103

Introduction.-The study of the decay of small perturbations has a long history in general relativity (GR). An increasingly precise knowledge of the quantitative form of the decay of fluctuations is required to advance our understanding of gravitation, from the interpretation of gravitational wave data to the study of fundamental questions like the deterministic character of GR.

The well-known appearance of Cauchy horizons in astrophysically relevant solutions of Einstein's equations signals a potential breakdown of determinism within GR - the future history of any observer that crosses such a horizon cannot be determined using the Einstein field equations and the initial data. Nonetheless, in the context of black hole $(\mathrm{BH})$ spacetimes, one expects that perturbations of the exterior region might be infinitely amplified by a blueshift mechanism, turning a Cauchy horizon in the $\mathrm{BH}$ interior into a singularity (terminal boundary) beyond which the field equations cease to make sense. Penrose's Strong Cosmic Censorship (SCC) conjecture substantiates this expectation.

On the other hand, astrophysical BHs are expected to be stable due to perturbation damping mechanisms acting in the exterior region. Therefore, whether or not SCC holds true hinges to a large extent on a delicate competition between the decay of perturbations in the exterior region and their (blueshift) amplification in the $\mathrm{BH}$ interior. For concreteness, let $\Phi$ be a linear scalar perturbation (i.e., a solution of the wave equation) on a fixed subextremal Reissner-Nordström (RN), asymptotically flat or de Sitter (dS) $\mathrm{BH}$, with cosmological constant $\Lambda \geq 0$. Regardless of the sign of $\Lambda$, in standard coordinates, the blueshift effect leads to an exponential divergence governed by the surface gravity of the Cauchy horizon $\kappa_{-}$.

Now the decay of perturbations depends crucially on the sign of $\Lambda$. For $\Lambda=0, \Phi$ satisfies an inverse power law decay [1-3] which is expected to be sufficient to stabilize the $\mathrm{BH}$ while weak enough to be outweighed by the blueshift amplification. Various results [4-8] then suggest that, in this case, the Cauchy horizon will become, upon perturbation, a mass inflation singularity, strong enough to impose the breakdown of the field equations.

For $\Lambda>0$, the situation changes dramatically. In fact, it has been shown rigorously that, for some $\Phi_{0} \in \mathbb{C}$ [9-12],

$$
\left|\Phi-\Phi_{0}\right| \leq C e^{-\alpha t},
$$

with $\alpha$ the spectral gap, i.e., the size of the quasinormal mode (QNM)-free strip below the real axis. Moreover, this result also holds for nonlinear coupled gravitational and electromagnetic perturbations of Kerr-Newman-dS (with small angular momentum) $[13,14]$. This is alarming as the exponential decay of perturbations might now be enough to counterbalance the blueshift amplification. As a consequence the fate of the Cauchy horizon now depends on the 
relation between $\alpha$ and $\kappa_{-}$. Will it still, upon perturbation, become a "strong enough" singularity in order to uphold SCC?

A convenient way to measure the strength of such a (Cauchy horizon) singularity is in terms of the regularity of the spacetime metric extensions it allows [15-17]. For instance, mass inflation is related to inextendibility in (the Sobolev space) $H^{1}$ which turns out to be enough to guarantee the nonexistence of extensions as (weak) solutions of the Einstein equations [18], i.e., the complete breakdown of the field equations.

As a proxy for extendibility of the metric itself, we will focus on the extendibility of a linear scalar perturbation. On a fixed RNdS, the results in Ref. [19] (compare with Ref. [20]) show that $\Phi$ extends to the Cauchy horizon with regularity at least

$$
H^{1 / 2+\beta}, \quad \beta \equiv \alpha / \kappa_{-} .
$$

Now the nonlinear analysis of Refs. $[13,14,21,22]$ suggests that the metric will have similar extendibility properties as the scalar field. It is then tempting to conjecture, as was done before in Refs. [6,23,24]: if there exists a parameter range for which $\beta>1 / 2$ then the corresponding (cosmological) $\mathrm{BH}$ spacetimes should be extendible beyond the Cauchy horizon with metric in $H^{1}$. Even more strikingly, one may be able to realize some of the previous extensions as weak solutions of the Einstein equations. This would correspond to a severe failure of SCC, in the presence of a positive cosmological constant. (The construction of bounded Hawking mass solutions of the Einstein-Maxwell-scalar field system with a cosmological constant allowing for $H^{1}$ extensions beyond the Cauchy horizon was carried out in [21]; these results use the stronger requirement $\beta>7 / 9$, but we expect $\beta>1 / 2$ to be sharp).

It is also important to note that if $\beta$ is allowed to exceed unity then (by Sobolev embedding) the scalar field extends in $C^{1}$; the coupling to gravity should then lead to the existence of solutions with bounded Ricci curvature. Moreover, for spherically symmetric self-gravitating scalar fields, the control of both the Hawking mass and the gradient of the field is enough to control the Kretschmann scalar [16]. We will, henceforth, relate $\beta<1$ to the blow up of curvature components.

At this moment, to understand the severeness of the consequences of the previous discussion, what we are most lacking is an understanding of how the decay rate of perturbations $\alpha$ is related to $\kappa_{-}$. Since $\alpha$ is the spectral gap, this can be achieved by the computation of the QNMs of RNdS BHs. The purpose of this work is to perform a comprehensive study of such modes and to discuss possible implications for SCC by determining $\beta$ throughout the parameter space of RNdS spacetimes.

Setting.-We focus on charged BHs in de Sitter spacetimes, the RNdS solutions. In Schwarzschild-like coordinates, the metric reads

$$
d s^{2}=-F(r) d t^{2}+\frac{d r^{2}}{F(r)}+r^{2}\left(d \theta^{2}+\sin ^{2} \theta d \phi^{2}\right),
$$

where $F(r)=1-2 M r^{-1}+Q^{2} r^{-2}-\Lambda r^{2} / 3 . M, Q$ are the $\mathrm{BH}$ mass and charge and $\Lambda$ is the cosmological constant. The surface gravity of each horizon is then

$$
\kappa_{*}=\frac{1}{2}\left|F^{\prime}\left(r_{*}\right)\right|, \quad * \in\{-,+, c\},
$$

where $r_{-}<r_{+}<r_{c}$ are the Cauchy horizon, event horizon and cosmological horizon radius.

A minimally coupled scalar field on a RNdS background with harmonic time dependence can be expanded in terms of spherical harmonics,

$$
\sum_{l m} \frac{\Phi_{l m}(r)}{r} Y_{l m}(\theta, \phi) e^{-i \omega t}
$$

Dropping the subscripts on the radial functions, they satisfy the equation

$$
\frac{d^{2} \Phi}{d r_{*}^{2}}+\left(\omega^{2}-V_{l}(r)\right) \Phi=0,
$$

where we introduced the tortoise coordinate $d r_{*}=d r / F$. The effective potential for scalar perturbations is

$$
V_{l}(r)=F(r)\left(\frac{l(l+1)}{r^{2}}+\frac{F^{\prime}(r)}{r}\right),
$$

where $l$ is an angular number, corresponding to the eigenvalue of the spherical harmonics.

We will be mostly interested in the characteristic frequencies of this spacetime, obtained by imposing the boundary conditions

$$
\Phi\left(r \rightarrow r_{+}\right) \sim e^{-i \omega r_{*}}, \quad \Phi\left(r \rightarrow r_{c}\right) \sim e^{i \omega r_{*}},
$$

which select a discrete set of frequencies $\omega_{\ln }$, called the QN frequencies [25]. They are characterized, for each $l$, by an integer $n \geq 0$ labeling the mode number. The fundamental mode $n=0$ corresponds, by definition, to the longest-lived mode, i.e., to the frequency with the smallest (in absolute value) imaginary part.

To determine the spectral gap $\alpha$, and, hence, the decay rate of perturbations, we will focus on the set of all modes $\omega_{\ln }$ [For $l=0$ there is a zero mode, corresponding to $\Phi_{0}$ in Eq. (1), which we ignore here.] and set

$$
\alpha \equiv \inf _{l n}\left\{-\operatorname{Im}\left(\omega_{\ln }\right)\right\}, \quad \beta \equiv \alpha / \kappa_{-} .
$$

We will henceforth drop the "In" subscripts to avoid cluttering. In previous works, we have used a variety of methods to compute the QNMs $[25,26]$. The results shown here were obtained mostly with the Mathematica package of Ref. [27] (based on methods developed in Ref. [28]), and checked in various cases with a variety of other methods $[25,26,29,30]$. 


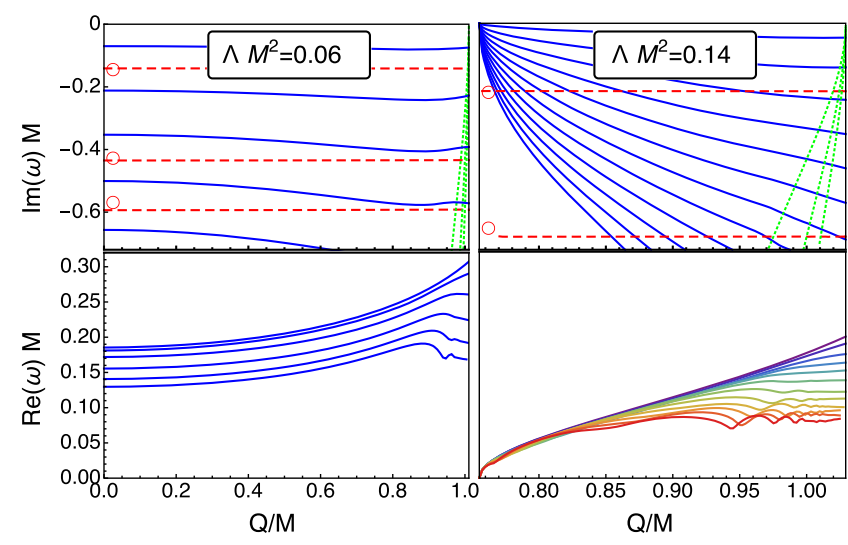

FIG. 1. Lowest lying quasinormal modes for $l=1$ and $\Lambda M^{2}=$ 0.06 (left) and 0.14 (right), as a function of $Q / M$. The top plots show the imaginary part, with dashed red lines corresponding to purely imaginary modes, and solid blue to complex, PS modes, whose real part is shown in the lower plots. The red circles in the top plots indicate the modes of empty de Sitter at the same $\Lambda$, which closely matches the first imaginary mode here, but lie increasingly less close to the higher modes. Near the extremal limit of maximal charge, another set of purely imaginary modes (dotted green lines) comes in from $-\infty$ and approaches 0 in the limit. Only a finite number of modes are shown, even though we expect infinitely many complex and extremal modes in the range shown.

QNMs of RNdS BHs: The three families.-Our results are summarized in Figs. 1-3 where one can distinguish three families of modes.

Photon sphere modes: Black holes and other sufficiently compact objects have trapping regions. Here, null particles can be trapped on circular unstable trajectories, defining the photon sphere. This region has a strong pull in the control of the decay of fluctuations and the spacetime's QNMs which have large frequency (i.e., large $|\operatorname{Re} \omega|$ ) [11,31-33]. For instance, the decay time scale is related to the instability time scale of null geodesics near the photon sphere. For BHs in de Sitter space, we do find a family of modes which can be traced back to the photon sphere. We refer to them as "photon sphere modes," or in short "PS" modes. These modes are depicted in blue (solid line) in Figs. 1-3. Different lines correspond to different overtones $n$; the fundamental mode is determined by the large $l$ limit (and $n=0$ ); we find that $l=10$ or $l=100$ provide good approximations of the imaginary parts of the dominating mode; note, however, that the real parts do not converge when $l \rightarrow \infty$. These modes are well described by a WKB approximation, and for very small cosmological constants they asymptote to the Schwarzschild BH QNMs [26].

For small values of the cosmological constant, PS modes are only weakly dependent on the $\mathrm{BH}$ charge. This is apparent from Fig. 1.

For $\Lambda M^{2}>1 / 9$ there is now a nonzero minimal charge, at which $r_{+}=r_{c}$. This limit is the charged Nariai BH and is shown as the blue dashed line in Fig. 2. The corresponding

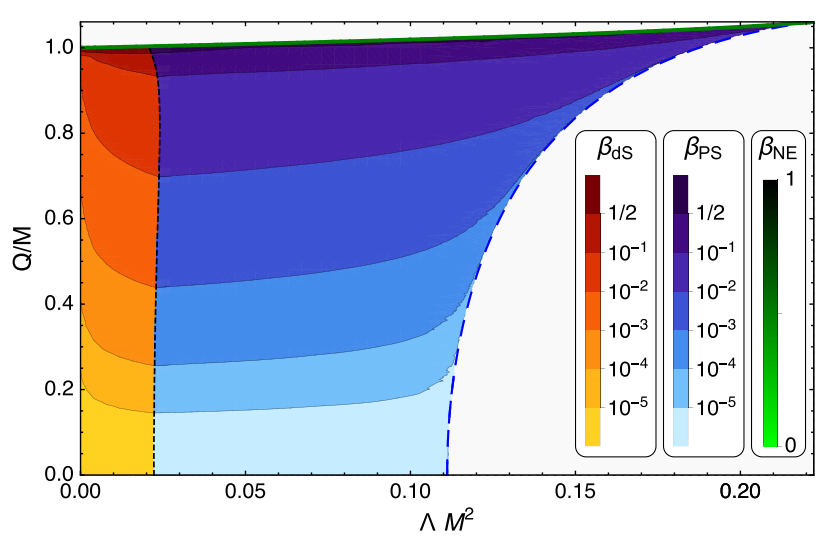

FIG. 2. Parameter space of the RNdS solutions, bounded by a line of extremal solutions of maximal charge where $r_{-}=r_{+}$on top, and for $\Lambda M^{2}>1 / 9$ a line of extremal solutions where $r_{c}=r_{+}$. In the physical region the value of $\beta$ is shown. For small $\Lambda M^{2}$ the dominant mode is the $l=1$ de Sitter mode, shown in shades of red. For larger $\Lambda M^{2}$ the dominant mode is the large $l$ complex, PS mode, here showing the $l=100 \mathrm{WKB}$ approximated mode in shades of blue. For very large $Q / M$ the $l=0$ extremal mode dominates. The green sliver on top where the NE mode dominates is merely indicative, the true numerical region is too small to be noticeable on these scales.

QNMs are also qualitatively different, as seen in Fig. 1. They, in fact, vanish in this limit, a result that can be established by solving the wave equation analytically to obtain (see Ref. [34] for the neutral case, we have generalized it to charged BHs, see Supplemental Material [35])

$$
\frac{\operatorname{Im}(\omega)}{\kappa_{+}}=-i\left(n+\frac{1}{2}\right)
$$

Note that the results presented here are enough to disprove a conjecture [36] that suggested that $\alpha$ should be equal to $\min \left\{\kappa_{+}, \kappa_{c}\right\}$. Such possibility is inconsistent with (10) and it is also straightforward to find other nonextremal parameters for which the WKB prediction yields smaller $\alpha$ 's (e.g., for $\Lambda M^{2}=0.1$ and $Q=0$ we have $\kappa_{+}=0.06759, \kappa_{c}=0.05249$, and $\alpha=0.03043$ ).

$d S$ modes: Note that solutions with purely imaginary $\omega$ exist in pure dS spacetime [37,38]

$$
\begin{gathered}
\omega_{0, \text { pure dS }} / \kappa_{c}^{\mathrm{dS}}=-i l, \\
\omega_{n \neq 0, \text { pure dS }} / \kappa_{c}^{\mathrm{dS}}=-i(l+n+1) .
\end{gathered}
$$

Our second family of modes, the (BH) dS modes, are deformations of the pure de Sitter modes (12); the dominant mode $(l=1, n=0)$ is almost identical and higher modes have increasingly larger deformations.

These modes are intriguing, in that they have a surprisingly weak dependence on the $\mathrm{BH}$ charge and seem to be described by the surface gravity $\kappa_{c}^{\mathrm{dS}}=\sqrt{\Lambda / 3}$ of the cosmological horizon of pure de Sitter, as opposed to that 


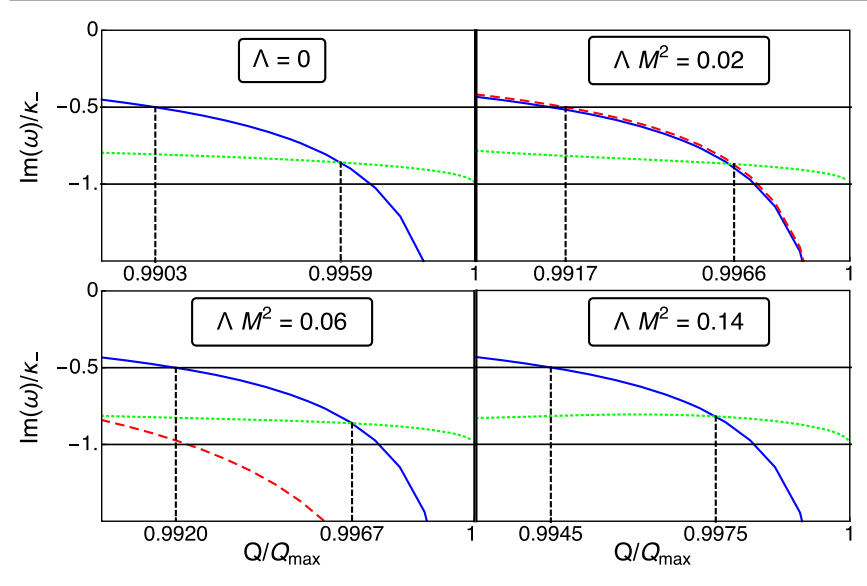

FIG. 3. Dominant modes of different types, showing the (nearly) dominant complex PS mode (blue, solid) at $l=10$, the dominant de Sitter mode (red, dotted) at $l=1$ and the dominant NE mode (green, dashed) at $l=0$. The two dashed vertical lines indicate the points where $\beta \equiv-\operatorname{Im}(\omega) / \kappa_{-}=1 / 2$ and where the NE becomes dominant. (Note that the value of $\beta$ is only relevant for $\Lambda>0$.)

of the cosmological horizon in the RNdS $\mathrm{BH}$ under consideration. This can, in principle, be explained by the fact that the accelerated expansion of the RNdS spacetimes is also governed by $\kappa_{c}^{\mathrm{dS}}[39,40]$.

This family has been seen in time evolutions [41] but, to the best of our knowledge, was only recently identified in the QNM calculation of neutral BH spacetimes [27]. Furthermore, our results indicate that as the $\mathrm{BH}$ "disappears" $\left(\Lambda M^{2} \rightarrow 0\right)$, these modes converge to the exact de Sitter modes (both the eigenvalue and the eigenfunction itself).

Near-extremal modes: Finally, in the limit that the Cauchy and event horizon radius approach each other, a third "near extremal" family, labeled as $\omega_{\mathrm{NE}}$, dominates the dynamics. In the extremal limit this family approaches

$$
\omega_{\mathrm{NE}}=-i(l+n+1) \kappa_{-}=-i(l+n+1) \kappa_{+},
$$

independently of $\Lambda$, as shown by our numerics. As indicated by Eq. (13), the dominant mode in this family is that for $l=0$, this remains true away from extremality.

In the asymptotically flat case, such modes seem to have been described analytically in Refs. [42-44]. Here we have shown numerically that such modes exist, and that they are in fact the limit of a new family of modes. It is unclear (but see Ref. [43]) if the NE family is a charged version of the zero-damping modes discussed recently in the context of rotating Kerr BHs [45]. It is also unclear if there is any relation between such long lived modes and the instability of exactly extremal geometries [46,47].

Maximizing $\beta$.- The dominating modes of the previous three families determine $\beta$, shown in Fig. 2 . Each family has a region in parameter space where it dominates over the other families. The dS family is dominant for "small" BHs (when $\Lambda M^{2} \lesssim 0.02$ ). In the opposite regime the PS modes are dominant. Notice that in the limit of minimal charge $\beta=0$, since $\kappa_{-}$remains finite while the imaginary parts of QNMs in the PS family approach 0 according to Eq. (10) (since $\kappa_{+} \rightarrow 0$ ).

More interesting is the other extremal limit, of maximal charge. In Fig. 2, the uppermost contours of the dS and PS families show a region where $\beta>1 / 2$.

Within this region as the charge is increased even further, the NE family becomes dominant. In Fig. 2 this is shown merely schematically, as the region is too small to plot on this scale, but it can already be seen in Fig. 1.

To see more clearly how $\beta$ behaves in the extremal limit we show 4 more constant $\Lambda M^{2}$ slices in Fig. 3. Here one sees clearly how above some value of the charge $\beta>1 / 2$, as dictated by either the de Sitter or the PS family. Increasing the charge further, $\beta$ would actually diverge if it were up to these two families ( $\omega M$ approaches a constant for both families, so $\omega / \kappa_{-}$diverges). However, the NE family takes over to prevent $\beta$ from becoming larger than 1 . Further details on these modes and on the maximum $\beta$ are shown in the Supplemental Material [35].

Conclusions. - The results in Refs. [13,14] show that the decay of small perturbations of de Sitter BHs is dictated by the spectral gap $\alpha$. At the same time, the linear analysis in Ref. [19] and the nonlinear analysis in Ref. [21] indicate that the size of $\beta \equiv \alpha / \kappa_{-}$controls the stability of Cauchy horizons and consequently the fate of the SCC conjecture. Recall that for the dynamics of the Einstein equations, and also for the destiny of observers, the blow up of curvature (related to $\beta<1$ ) per se is of little significance: it implies neither the breakdown of the field equations [48] nor the destruction of macroscopic observers [49]. In fact, a formulation of SCC in those terms is condemned to overlook relevant physical phenomena like impulsive gravitational waves or the formation of shocks in relativistic fluids. For those and other reasons, the modern formulation of SCC, which we privilege here, makes the stronger request $\beta<\frac{1}{2}$ in order to guarantee the breakdown of the field equation at the Cauchy horizon.

Here, by studying (linear) massless scalar fields and searching through the entire parameter space of subextremal and extremal RNdS spacetimes, we find ranges for which $\beta$ exceeds $1 / 2$ but, remarkably, it does not seem to be allowed, by the appearance of a new class of nearextremal modes, to exceed unity. This opens the perspective of having Cauchy horizons which, upon perturbation, can be seen as singular, by the divergence of curvature invariants, but nonetheless maintain enough regularity as to allow the field equations to determine (classically), in a highly nonunique way, the evolution of gravitation. This corresponds to a severe failure of determinism in GR. 
Finally, even though the BHs we considered are charged and not expected to occur in nature, we do expect very similar results to hold for BHs which are neutral but rotating, by the standard charge-angular momentum analogy, where near-extremal charge corresponds to fast rotating BHs. Given the nonzero cosmological constant and the existence of rapidly rotating $\mathrm{BHs}$ in our Universe [50-52], these results cannot be taken lightly.

V. C. is indebted to Kinki University in Osaka and to the Kobayashi-Maskawa Institute in Nagoya for hospitality, while the late stages of this work were being completed. V.C. and K. D. acknowledge financial support provided under the European Union's H2020 ERC Consolidator Grant "Matter and strong-field gravity: New frontiers in Einstein's theory" Grant agreement No. MaGRaTh646597. Research at Perimeter Institute is supported by the Government of Canada through Industry Canada and by the Province of Ontario through the Ministry of Economic Development \& Innovation. This project has received funding from the European Union's Horizon 2020 research and innovation programme under the Marie SklodowskaCurie Grant Agreement No. 690904. Part of this research was conducted during the time P. H. served as a Clay Research Fellow, supported by the Clay Mathematics Institute; P. H. also acknowledges support from the Adolph C. and Mary Sprague Miller Institute for Basic Research in Science, University of California Berkeley. The authors would like to acknowledge networking support by the COST Action GWverse CA16104. The authors thankfully acknowledge the computer resources, technical expertise and assistance provided by Sérgio Almeida at CENTRA/IST. Computations were performed at the cluster "Baltasar-Sete-Sóis," and supported by the MaGRaTh646597 ERC Consolidator Grant. J. L. C. acknowledges financial support provided by FCT/Portugal through UID/ MAT/04459/2013 and Grant (GPSEinstein) PTDC/MATANA/1275/2014. A. J. was supported by the Netherlands Organisation for Scientific Research (NWO) under VIDI Grant No. 680-47-518, and the Delta-Institute for Theoretical Physics (D-ITP) that is funded by the Dutch Ministry of Education, Culture and Science (OCW).

[1] R. H. Price, Phys. Rev. D 5, 2419 (1972).

[2] M. Dafermos, I. Rodnianski, and Y. Shlapentokh-Rothman, arXiv:1402.7034.

[3] Y. Angelopoulos, S. Aretakis, and D. Gajic, Adv. Math. 323, 529 (2018).

[4] E. Poisson and W. Israel, Phys. Rev. D 41, 1796 (1990).

[5] M. Dafermos, Commun. Pure Appl. Math. 58, 0445 (2005).

[6] M. Dafermos, Commun. Math. Phys. 332, 729 (2014).

[7] J. Luk and S.-J. Oh, arXiv:1702.05715.

[8] J. Luk and S.-J. Oh, arXiv:1702.05716.
[9] A. Sá Barreto and M. Zworski, Math. Res. Lett. 4, 103 (1997).

[10] J.-F. Bony and D. Häfner, Commun. Math. Phys. 282, 697 (2008).

[11] S. Dyatlov, Ann. Henri Poincare 13, 1101 (2012).

[12] S. Dyatlov, Commun. Math. Phys. 335, 1445 (2015).

[13] P. Hintz and A. Vasy, arXiv:1606.04014.

[14] P. Hintz, arXiv:1612.04489.

[15] A. Ori, Phys. Rev. D 61, 064016 (2000).

[16] J. L. Costa, P. M. Girão, J. Natário, and J. D. Silva, Ann. PDE 3, 8 (2017).

[17] J. Earman, Bangs, Crunches, Whimpers, and Shrieks (Oxford University Press, Oxford, 1995).

[18] D. Christodoulou, arXiv:0805.3880.

[19] P. Hintz and A. Vasy, J. Math. Phys. (N.Y.) 58, 081509 (2017).

[20] J. L. Costa and A. T. Franzen, Ann. Henri Poincaré 18, 3371 (2017).

[21] J. L. Costa, P. M. Girão, J. Natário, and J. D. Silva, arXiv: 1707.08975

[22] M. Dafermos and J. Luk, arXiv:1710.01722.

[23] K. Maeda, T. Torii, and M. Narita, Phys. Rev. D 61, 024020 (1999).

[24] J. L. Costa, P. M. Girão, J. Natário, and J. D. Silva, Classical Quantum Gravity 32, 015017 (2015).

[25] E. Berti, V. Cardoso, and A. O. Starinets, Classical Quantum Gravity 26, 163001 (2009).

[26] https://centra.tecnico.ulisboa.pt/network/grit/files/.

[27] A. Jansen, arXiv:1709.09178.

[28] O. J. C. Dias, P. Figueras, R. Monteiro, H. S. Reall, and J. E. Santos, J. High Energy Phys. 05 (2010) 076.

[29] K. Lin and W.-L. Qian, arXiv:1609.05948.

[30] S. Iyer and C. M. Will, Phys. Rev. D 35, 3621 (1987).

[31] V. Cardoso and P. Pani, Nat. Astron. 1, 586 (2017).

[32] V. Cardoso, A. S. Miranda, E. Berti, H. Witek, and V. T. Zanchin, Phys. Rev. D 79, 064016 (2009).

[33] S. Dyatlov and M. Zworski, Phys. Rev. D 88, 084037 (2013).

[34] V. Cardoso and J. P. S. Lemos, Phys. Rev. D 67, 084020 (2003).

[35] See Supplemental Material at http://link.aps.org/ supplemental/10.1103/PhysRevLett.120.031103 for analytic derivation, numerical checks and eigenfunction plot.

[36] P. R. Brady, I. G. Moss, and R. C. Myers, Phys. Rev. Lett. 80, 3432 (1998).

[37] A. Lopez-Ortega, Gen. Relativ. Gravit. 44, 2387 (2012).

[38] A. Vasy, Adv. Math. 223, 49 (2010).

[39] D. R. Brill and S. A. Hayward, Classical Quantum Gravity 11, 359 (1994).

[40] A. D. Rendall, Ann. Henri Poincare 5, 1041 (2004).

[41] P. R. Brady, C. M. Chambers, W. G. Laarakkers, and E. Poisson, Phys. Rev. D 60, 064003 (1999).

[42] Y.-W. Kim, Y. S. Myung, and Y.-J. Park, Eur. Phys. J. C 73, 2440 (2013).

[43] A. Zimmerman and Z. Mark, Phys. Rev. D 93, 044033 (2016); 93, 089905 (2016).

[44] S. Hod, Eur. Phys. J. C 77, 351 (2017).

[45] M. Richartz, C. A. R. Herdeiro, and E. Berti, Phys. Rev. D 96, 044034 (2017).

[46] S. Aretakis, Adv. Theor. Math. Phys. 19, 507 (2015). 
[47] M. Casals, S. E. Gralla, and P. Zimmerman, Phys. Rev. D 94, 064003 (2016).

[48] S. Klainerman, I. Rodnianski, and J. Szeftel, Inventiones Mathematicae 202, 91 (2015).

[49] A. Ori, Phys. Rev. Lett. 67, 789 (1991).

[50] L. W. Brenneman, C. S. Reynolds, M. A. Nowak, R. C. Reis, M. Trippe, A. C. Fabian, K. Iwasawa, J. C. Lee, J. M. Miller, R. F. Mushotzky, K. Nandra, and M. Volonteri, Astrophys. J. 736, 103 (2011).
[51] M. Middleton, Black hole spin: Theory and observation, in Astrophysics of Black Holes: From Fundamental Aspects to Latest Developments, edited by C. Bambi (Springer, Berlin, Heidelberg, 2016), p. 99.

[52] G. Risaliti, F. Harrison, K. Madsen, D. Walton, S. Boggs, F. Christensen, W. Craig, B. Grefenstette, C. Hailey, E. Nardini et al., Nature (London) 494, 449 (2013). 